\title{
Effects of JPEG and JPEG2K Standard Algorithms on Compression of Laser Speckle Images: Image Quality and on a Clinical Parameter Accuracy
}

\author{
AL-Nuaimi Bashar, Taha Mohammed Hasan, Zainab Mohammed Ali Al-Azzawi and \\ Jumana Waleed \\ Department of Computer Science, College of Science, University of Diyala, Diyala, Iraq \\ al-nuaimibashar@sciences.uodiyala.
}

\begin{abstract}
Laser Speckle Contrast Imaging (LSCI) is a full-field optical method to monitor microvascular blood flow. This technique provides images with excellent spatial and temporal resolution information from which blood cells velocity values can be computed. Compression offers a means to reduce the space storage and increase the speed of transmission. The goal of this research is to analysis the effect of laser speckle perfusion images compression with standard algorithms (lossy and lossless). In this study, we selected 10 sequences of 60 images of LSCI. Standard compression algorithms JPEG and JPEG2k deal with integer pixel values (pixels coded on 1 or 2 bytes) which is not the case for LSCI data. Therefore, the aim to test a standard algorithm on 10 LSCI recording. Experimental results are evaluated in terms of compression ratio and Peak Signal to Noise Ratio (PSNR). For this purpose, we suggest to process laser speckle contrast images by calculate the differences in velocity values which is used as a clinical quality measurement by compute from images obtained after a 8 bits integer conversion by lossless and lossy version. This study demonstrate the ability to compress LSCI data with a standard method with high average compression ratio for perfusion image and good image quality.
\end{abstract}

Key words: LSCI, JPEG, JPEG2K, image compression, quality assessment, standard method

\section{INTRODUCTION}

Laser Speckle Contrast Imaging (LSCI) is a recent optical technique used in the biomedical field to monitor microvascular blood flow (microvascular perfusion) (Chen et al., 2016). This imaging modality has the advantage of being a contactless technique and it leads to perfusion images with high spatial and temporal resolutions (Boas and Dunn, 2010). Moreover, from LSCI data, velocity of microvascular moving scatterers (mainly red blood cells from the microcirculation) can be evaluated when assumptions on the scatterers velocity distribution are made. A Lorentzian velocity distribution is often chosen in the literature (Briers and Webster, 1996). However, the drawback of LSCI is that it leads to large data: perfusion images can be acquired with a sampling frequency of 16 images/s (or even higher) and each perfusion image may have a size of about $250 \times 140$ pixels $^{2}$ when a zone of $9.3 \times 4.0 \mathrm{~cm}^{2}$ is studied. Moreover, if each pixel is coded with 8 bits, a $40 \mathrm{~min}$ recordings may require a space of $10 \mathrm{~GB}$. The question that therefore, emerges is how can we store and transmit such large data.

A compression process aims at creating an alternative form for the information representation to reduce the necessary storage capacity and to increase the transmission bandwidth (Kocsis et al., 2003). When applied on medical images as laser speckle contrast images, the compression decompression procedures should lead to data that are still exploitable by the clinicians. The challenge is therefore to produce a high compression ratio while keeping the diagnosis performances.

This raises the question of how to store and transmit such large quantity of data. By reducing the value of data, compression offers an effective solution and present an economical alternative to the increasing measuring stage capacity. The challenge of LSCI compression is to find the best possible comparative between $\mathrm{CR}$ and reconstructed image quality. As the compression process aimed the creation of an intermediary form of the information (which is more compact), the use of standard compression is particularly important in medical imaging in order to meet the needs for interoperability and life. Span which arise from the healthcare sector. So for this study, we evaluated two standard image compression methods.

JPEG and JPEG2K: The goal of our research is to study if JPEG and JPEG2k algorithms can be of interest for laser speckle contrast images (storage and transmission). To answer this question, we will apply the two compression

Corresponding Author: AL-Nuaimi Bashar, Department of Computer Science, College of Science, University of Diyala, Diyala, Iraq, al-nuaimi bashar@sciences.uodiyala.edu.iq 
algorithms on stack of LSCI perfusion images recorded in the forearm skin of 10 subjects. The quality of the decompressed images will be analyzed by comparing blood cells velocity values computed from the decompressed images with the ones computed from the original stack of images.

\section{MATERIALS AND METHODS}

Laser speckle contrast imaging: LSCI is now commonly used in clinical research to monitor microvascular blood flow in tissues as retina, skin and brain (Dunn et al., 2001). The principle of the technique is the following: a coherent light (laser light) illuminates the tissue under study. The backscattered light forms an interference pattern (known as a speckle pattern) on a detector that is a video camera. Due to the movement of scattering particles (as red blood cells) in the tissue, the speckle patterns fluctuate in intensity. When the exposure time of the camera is longer than the time scale of the speckle intensity fluctuations, the camera integrates the intensity variations which results in a blurring of the speckle pattern. The degree of blurring is quantified by the spatial speckle contrast $\mathrm{K}$ that is defined as the ratio of the standard deviation $\sigma$ of the intensity I to the mean pixel Intensity (I) of the speckle pattern in a square window of $\mathrm{N}^{2}$ pixels (Briers and Webster, 1996):

$$
\mathrm{K}=\frac{\sigma}{\langle\mathrm{I}\rangle}=\frac{\sqrt{\left\langle\mathrm{I}^{2}\right\rangle-\langle\mathrm{I}\rangle^{2}}}{\langle\mathrm{I}\rangle}
$$

To obtain a two-dimensional contrast image, the square window is moved along the raw speckle image. The LSCI perfusion image is then computed from the inverse of the contrast value in each pixel (see an example of a LSCI perfusion image in Fig. 1.

From laser speckle contrast images, velocity of moving scatterers from the microcirculation (mainly red blood cells of the microcirculation) can be computed when a velocity profile is assumed for moving scatterers. If this profile is assumed to follow Lorentzian statistics we have (Boas and Dunn, 2010):

$$
\mathrm{K}=\beta\left[\frac{\tau_{\mathrm{c}}}{\mathrm{T}}+\frac{\tau_{\mathrm{c}}^{2}}{2 \mathrm{~T}^{2}}\left(\exp \left(-\frac{2 \mathrm{~T}}{\tau_{\mathrm{c}}}\right)-1\right)\right]
$$

Where:

$\mathrm{T}=$ The exposure time of the camera

$\tau_{c}=$ The correlation time of intensity fluctuations.

The relation between correlation time $\tau_{c}$ and the velocity of moving scatterers $v_{c}$ is given by (Briers and Webster, 1996):

$$
v_{\mathrm{c}}=\frac{\lambda}{2 \pi \tau_{\mathrm{c}}}
$$

where, $\lambda$ is the laser wavelength.

Lossless and lossy compression-coding techniques: Many types of compression methods have been developed. These methods fall into two broad types, lossless and lossy algorithms. A lossless compression technique allowed the original data to be perfect by reconstructed from the compressed data. Lossless compression technique exploits coding redundancy to decrease the number of bits needed to represent an image (Kivijarvi et al., 1998). These algorithms are called reversible compression techniques. Compression rates for lossless methods vary but typically are around 2:1-3:1 (Georgiev et al., 2013; Liu et al., 2017; Elhannachi et al., 2017).

By contrast, lossy image compression schemes permit re-construction only of an approximation of the original data to improve the compression ratio (Mateika and Martavicius, 2007). These techniques allow high compression rates that depending on the compression quality and the image features. At high quality levels 10:1-20:1 (Ukrit et al., 2011).

Standard compression algorithms allow meeting the needs for interoperability and life-span which arise from the health care sector (Liu et al., 2017). The standard algorithms, Discrete Cosine Transform (DCT) and the Discrete Wavelet Transforms (DWT) are widely used for image and video compression. These two standard methods provide a higher image compression to reduce the cost of storage capacity and higher transmission speed (Rabbani and Joshi, 2002).

JPEG and JPEG2K are two standard image compression algorithms (Kim et al., 2010). JPEG that has been established by the (Joint Photographic Experts Group) has officially been accepted as an international standard in 1992. It is a lossy image compression method (Wallace, 1991). JPEG2k is another standard image compression algorithm that produces higher quality images compared to JPEG for a same compression ratio. Moreover, JPE2k offers lossy and lossless compressions in the same file stream and also spatial scalability with excellent compression performance (Przelaskowski , 2004).

Compression assessment: Measuring the quality of the image is a complex and hard process, since, human opinion is affected by physical parameters. Many methods are offered for image quality measuring but none of it is deliberate to be perfect for measuring quality. Image quality measurement plays an important role in the field of image processing (Kocsis et al., 2003). 
The losses in the image data usually correlated to the compressed images may be suitable or not depending on some predefined accepted level. Fidelity measures are often used to measure the amount of information losses produced by performing certain coding algorithms. Among the most commonly used is the Mean Square Error (MSE) test, defined as:

$$
\mathrm{MSE}=\frac{1}{\mathrm{~N} \times \mathrm{M}} \sum_{\mathrm{y}=0}^{\mathrm{N}-1} \sum_{\mathrm{x}=0}^{\mathrm{M}-1}[\mathrm{O}(\mathrm{x}, \mathrm{y})-\mathrm{R}(\mathrm{x}, \mathrm{y})]^{2}
$$

Where:

$$
\begin{aligned}
& \mathrm{O}=\text { Refers to the original image } \\
& \mathrm{R}=\text { The decoded image } \\
& \mathrm{N} \times \mathrm{M}=\text { Pixels is the image size }
\end{aligned}
$$

The most commonly used as fidelity measurement is Peak Signal to Noise Ratio (PSNR) is an expression for the ratio between the maximum possible value (power) of a signal and the power of distorting noise that affects the quality of its representation. PSNR is usually expressed in terms of the logarithmic decibel scale (Wang et al., 2004). The PSNR can be signified in decibel units $(\mathrm{dB})$ as:

$$
\text { PSNR }=10 \log _{10}\left[\frac{(\text { Max value-Min value })^{2}}{\text { MSE }}\right]
$$

It is well known that the objective measure like PSNR is not a good pointer of subjective quality. Specifically at small PSNR values and high compression ratios (Gonzalez et al., 2009). As mentioned above, velocity of moving particles form LSCI data can be calculated using Eq. 2: (Briers, 2007; Cho et al., 2016; Dunn, 2012). We have therefore, proposed the velocity of moving scatterers as a clinical quality measurement. Thereby, mean velocity difference was computed for original and reconstructed image.

$$
\text { Mean differences velocity }=\mathrm{E}\left\{\left[\mathrm{V}^{\prime}(\mathrm{i})-\mathrm{V}(\mathrm{i})\right]\right\}
$$

where, in $\mathrm{V}^{\prime}$ and $\mathrm{V}$ refers to the original and decoded velocity, respectively. Each of size (i), i: is a number of image while referring to expectation or average value of velocities.

The proposed compression scheme: In this study, we studied the ability to compress LSCI data with two major compression algorithms: JPEG and JPEG2k for both their lossless and lossy versions.

The data were recorded with a frequency sampling of $18 \mathrm{~Hz}$ on the arm of 10 healthy people. Four successive frames are shown in Fig. 1. We have selected 60 images
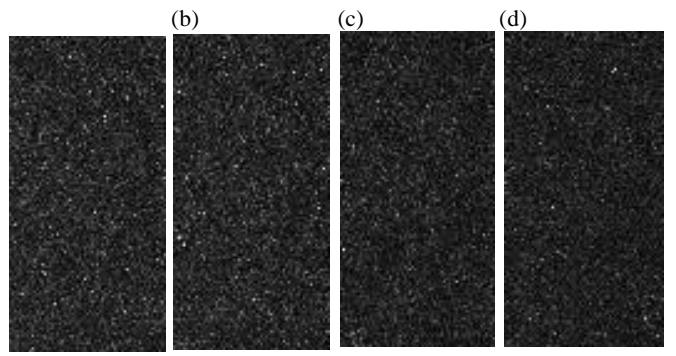

Fig. 1: Four successive LSCI perfusion images: a) Im1; b) $\operatorname{Im} 2 ;$ c) $\operatorname{Im} 3$ and d) $\operatorname{Im} 4$
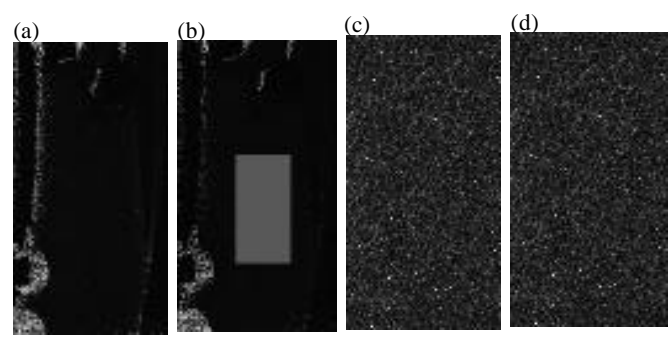

Fig. 2: Original ROI for perfusion image: a) Original frame; b) Original frame $+\mathrm{ROI}$; c) $\mathrm{ROI}$ frame and d) ROI integer frame

for each sample. For each image in the sequence, a Region of Interest (ROI) with a size of $128 \times 64$ pixels was selected as shown in Fig. 2. The proposed test was implemented using standard function (imwrite) in MATLAB (Gonzalez et al., 2009; Marques, 2011).

The second step is mapping a pixel value to an integer value. With convert the original float values into integer 8-bits unsigned image value. However, standard algorithms such as JPEG and JPEG2k in their lossless and lossy versions, deal with integer pixel values only (pixels coded on 1 or 2 bytes) which is not the case for LSCI data. So, we converted the original LSCI float pixel values into integer values. For a brief view, we can summarized the processing steps of of our study as the following. For lossless compression method, we compared $\mathrm{CR}$ and effect of integer conversion on PSNR and error on velocity for 8 and 16 bits calculating.

For lossy method is only with 8 bits which JPEG method deal with 8 bits only. Thereby, evaluate their efficiency in terms of Compression Ratio (CR). We studied the effects of these algorithms on the quality of reconstructed images in terms of induced pixel value degradations quantified with PSNR and flow distortion: flow is a clinical parameter calculated from perfusion sequence: 
- Defined CR with JPEG by fixing the quality factor from 10-100

- Use these CR as input parameters for JPEG2K to compare effect of compression for JPEG and JPEG2K

- Evaluate and compare $\mathrm{CR}$ and effect of integer conversion on PSNR. Furthermore, calculate the error on velocity for 8 bits for each quality factor.

\section{RESULTS AND DISCUSSION}

The results obtained for lossless version (JPEG2k and JPEG) are shown in Fig. 3. Figure 4 presents our new clinical quality parameter by calculating the differences in velocities values. These values were computed from images obtained after a 8 and 16 bits integer conversion. For lossy compression method, Fig. 5 present the relation between CR and PSNR after a 8 bits integer conversion for JPEG and JPEG2k. The relation between the compression ratio and the mean difference of velocities values are shown in Fig. 6.

This study is the first analysis on laser speckle images compression. The purpose of this study was to evaluate and compare the results of JPEG and JPEG2k for laser speckle contrast image compression. The expanded of this study of laser speckle contrast image compression with a the standard method by using speckle pattern velocity as a clinical quality parameter by calculating the mean difference velocities between the original image and reconstruction image. Image compression and degradation depend on image information which was confirmed by the quantitative results of this study and is in accord with the coding process. Various compression ratios were applied on perfusion image with different 10 samples to compare effect of compression for JPEG and JPEG2k.

The lossless compression efficiency of the reversible standard algorithm are reported in. The results obtained after compressed the perfusion image by the JPEG $2 \mathrm{k}$ and JPEG ( 8 bits) show approximately similar results for CR and PSNR values. This is true for all samples.

The significant results of velocity measure that used as clinical quality assessment are shown. The difference velocity value for perfusion image was obtained after encoding-decoding is approximately insignificant different compared to the original image velocity. These results verified the lossless technique provides advantages that LSCI images can be transferred effectively and are reconstructed perfectly.

Figure present the relation between the $\mathrm{CR}$ and PSNR for lossy version of JPEG and JPEG2k, respectively. For these Fig. 10 samples have been studied for each method. From these two figure, we can observe that the JPEG method is demonstrated a higher values of PSNR for

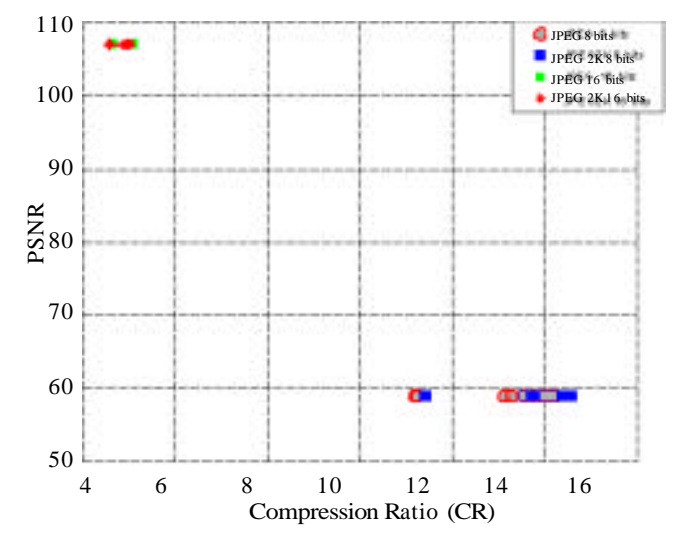

Fig. 3: Perfusion image compression by JPEG and JPEG2k 8 and 16 bits (lossless) for 10 samples are studied

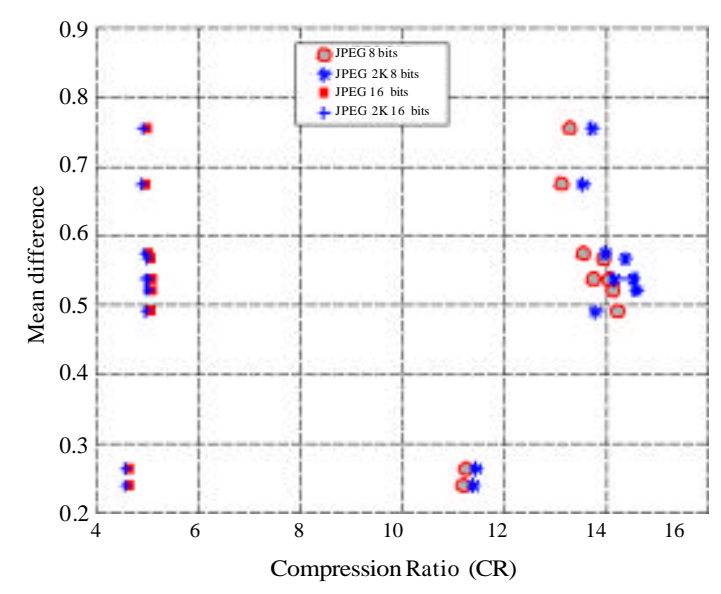

Fig. 4: Effect of 8 and 16 bits (lossless) conversion on perfusion original values and velocity values. The range of original values, the range of the difference and mean \pm standard deviation of the difference were calculated on a 60 image recording. Furthermore, 10 samples are studied

lower bit rates (bpp). JPEG is higher approximately by $2 \mathrm{~dB}$ compared to the JPEG2k results. The goal of CR is to compared the results of different samples of perfusion images. The JPEG $2 \mathrm{k}$ produces slightly better $\mathrm{CR}$ values compared to JPEG. For JPEG and JPEG2k lossy version, the proposed parameter of mean difference velocities as a function of $\mathrm{CR}$ is shown in, respectively. For these two Fig. 10 samples are studied. Furthermore, different quality of compression ratio is calculated. From these two figures, JPEG2k demonstrated more efficient mean velocities differences as well as a higher correlation values compared to JPEG2K that is true for all 10 samples. 


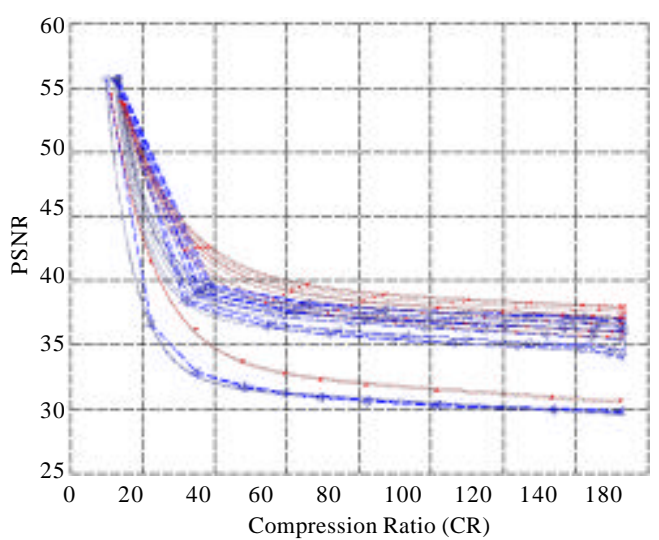

Fig. 5: The relation between CR and PSNR values of JPEG 8 bit lossy version. Each curve with represent one sample of 10 samples. Red curves refer to JPEG and blue curves refer to JPEG2K. Perfusion image compression by JPEG2000 (lossy)-exponetial-2 approximation

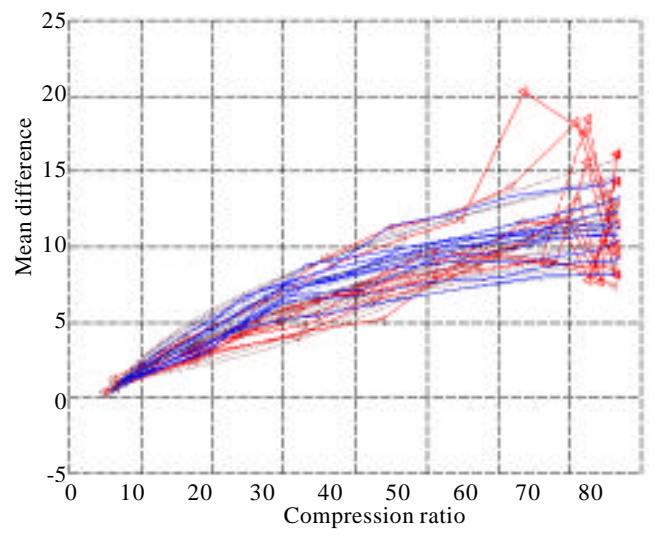

Fig. 6: The mean difference velocities versus the compression ratio for original perfusion image and reconstructed image after JPEG and JPEG2K 8 bits lossy compression. Red ( $-1>$ ) curve refer to JPEG and blue $(\bullet \bullet)$ curve refer to JPEG2K

\section{CONCLUSION}

In this study we proposed to use the speckle pattern velocity as a clinical quality measurement to validate our results that providing ensure that can be compressed LSCI without insignificant change on features of original image value with good average compression ratio and better image quality for perfusion image. These results are encouraging for going ahead in applying standard compression algorithms on LSCI data.

\section{REFERENCES}

Boas, D.A. and A.K. Dunn, 2010. Laser speckle contrast imaging in biomedical optics. J. Biomed. Opt., 15: $1-12$.

Briers, J.D. and S. Webster, 1996. Laser speckle contrast analysis (LASCA): A nonscanning, full-field technique for monitoring capillary blood flow. J. Biomed. Opt., 1: 174-179.

Briers, J.D., 2007. Laser speckle contrast imaging for measuring blood flow. Opt. Appl., 37: 139-152.

Chen, D., J. Ren, Y. Wang, H. Zhao and B. Li et al., 2016. Relationship between the blood perfusion values determined by laser speckle imaging and laser doppler imaging in normal skin and port wine stains. Photodiagn. Photodyn. Ther., 13: 1-9.

Cho, A., C. Yeon, D. Kim and E. Chung, 2016. Laser speckle contrast imaging for measuring cerebral blood flow changes caused by electrical sensory stimulation. J. Opt. Soc. Korea, 20: 88-93.

Dunn, A.K., 2012. Laser speckle contrast imaging of cerebral blood flow. Ann. Biomed. Eng., 40: 367-377.

Dunn, A.K., H. Bolay, M.A. Moskowitz and D.A. Boas, 2001. Dynamic imaging of cerebral blood flow using laser speckle. J. Cereb. Blood Flow Metab., 21: 195-201.

Elhannachi, S.A., N. Benamrane and T.A. Abdelmalik, 2017. Adaptive medical image compression based on lossy and lossless embedded zerotree methods. J. Inf. Proc. Syst., 13: 40-56.

Georgiev, V.T., A.N. Karahaliou, S.G. Skiadopoulos, N.S. Arikidis and A.D. Kazantzi et al., 2013. Quantitative visually lossless compression ratio determination of JPEG2000 in digitized mammograms. J. Digital Imaging, 26: 427-439.

Gonzalez, R.C., R.E. Woods and S.L. Eddins, 2009. Digital Image Processing Using MATLAB. 2nd Edn., Tata McGraw-Hill Education, New Delhi, India, ISBN:9781259084072, Pages: 826.

Kim, D.H., H.J. Kim, C.L. Lee, H.M. Cho and H.S. Park et al., 2010. Comparison and evaluation of JPEG and JPEG2000 in medical images for CR (Computed Radiography). J. Korean Phys. Soc., 56: 856-862.

Kivijarvi, J., T. Ojala, T. Kaukoranta, A. Kuba and L. Nyul et al., 1998. A comparison of lossless compression methods for medical images. Comput. Med. Imag. Graph., 22: 323-339.

Kocsis, O., L. Costardidou, L. Varaki, C. Kalogeroulou and G. Panayiotakis, 2003. Compression assessment based on medical image quality concepts using computer generated test images. Comput. Methods Programs Biomed., 71: 105-115. 
Liu, F., M. Hernandez-Cabronero, V. Sanchez, M.W. Marcellin and A. Bilgin, 2017. The current role of image compression standards in medical imaging. Inf., 8: 1-26.

Marques, O., 2011. Practical Image and Video Processing using MATLAB. Wiley Publishing Company, Hoboken, New Jersey, USA., ISBN:9781118093474, Pages: 696.

Mateika, D., R. Martavicius, 2007. Analysis of the compression ratio and quality in medical images. Inf. Technol. Control., 35: 419-423.

Przelaskowski, A., 2004. The JPEG2000 standard for medical image applications. Task Quart., 8: 147-158.
Rabbani, M. and R. Joshi, 2002. An overview of the JPEG 2000 still image compression standard. Signal Process. Image Commun., 17: 3-48.

Ukrit, M.F., A. Umamageswari and D.G.R. Suresh, 2011. A survey on lossless compression for medical images. Int. J. Comput. Appl., 31: 47-50.

Wallace, G.K., 1991. The JPEG still picture compression standard. Commun. ACM., 34: 30-44.

Wang, Z., A.C. Bovik, H.R. Sheikh and E.P. Simoncelli, 2004. Image quality assessment: From error visibility to structural similarity. IEEE Trans. Image Process., 13: $600-612$. 